\title{
Identification and characterization of human non-coding RNAs with tissue specific expression
}

Yasnory T. F. Sasaki ${ }^{a}$, Miho Sano ${ }^{a}, c$, Takashi Ideue ${ }^{a, c}$, Taishin $\mathrm{Kin}^{\mathrm{b}}$, Kiyo shi Asai ${ }^{\mathrm{b}, \mathrm{e}}$ and Tetsuro Hirose $\mathrm{e}^{\mathrm{a}, \mathrm{d} *}$

${ }^{\mathrm{a}}$ Functional RNA Research Team, Biological Information Research Center, ${ }^{\mathrm{b}}$ Mathematical Modeling Team, Computational Biology Research Center, National Institute of Advanced Industrial Science and Technology (AIST) and 'Japan Biological Informatics Consortium (JBIC), Tokyo 135-0064, Japan, 'PRESTO, Japan Science and Technology Agency, Saitama 332-0012, Japan, ' Graduate School of Frontier Sciences, University of Tokyo, Chiba 277-8583, Japan

*Corresponding author. Fax: +81-3-3599-8579.

E-mail address: tehirose@jbirc.aist.go.jp (T. Hirose) 


\section{Abstract}

We have examined the expression profile of selected non-coding RNAs (ncRNAs) in 11 human tissues. Among 5489 full-length cDNA clones annotated as non-protein-coding transcripts in the H-Invitational database, we chose 150 clones for further analysis based on their gene structure and EST information. Expression profiling using quantitative RT-PCR and Northern blot hybridization revealed that the majority of the selected ncRNAs exhibited tissue specificity: $\underline{67 \%}$ are predominantly expressed in a restricted subset of tissues. The absolute quantification of representative ncRNAs revealed that the majority of ncRNAs are expressed as low abundance transcripts. A comparative genomic analysis revealed that only $\underline{27 \%}$ of the selected ncRNAs have mouse counterparts. Since the expression patterns of the human ncRNAs having no mouse counterparts remain to be similar to tho se of the mouse ncRNAs, the expression patterns of the selected ncRNAs may be conserved between human and mouse.

Keywords: non-coding RNA; H-Invitational; expression profile; quantitative RT-PCR; northern blot hybridization

Post-genomic transcripto me analysis has revealed the existence of large numbers of transcripts that lack protein-coding potential and are therefore expected to exist as non-protein-coding RNAs [1,2, and refs therein]. Since this transcriptome analysis is based on sequencing of a full-length cDNA library constructed from RNA molecules possessing both a 5' cap structure and 3' poly $(A)$ tail, these ncRNAs have mRNA-like features at their termini. Whether all these transcripts are functional, independent transcription units or just experimental artifacts derived from truncated mRNAs is still in dispute. However, a number of these mRNA-like ncRNAs appear to play pivotal roles in mammalian cells, including transcriptional regulation, genomic imprinting, and protein transport $[3,4,5]$. Such evidence raises the intriguing possibility that ncRNAs play an important role in controlling various aspects of gene expression [1, 2, and refs therein].

The primary dataset of the human transcriptome is available in the $\mathrm{H}$-Invitational database (H-InvDB, http://www.h-invitational.jp). In the primary dataset from 2002, 1377 transcripts in which the longest ORF is less than 80 amino acids were isolated from 21,037 human full-length cDNAs. The transcripts in the H-InvDB have been classified based on their overlap with other transcripts, and the features of their $3^{\prime}$ end, as well as the 
existence of any corresponding EST clones. The putative ncRNAs include transcripts that are at a distance of $>5 \mathrm{~kb}$ from neighboring genes and are found in at least one EST database, and are thus likely to be genuine ncRNAs.

Here, we investigated the expression profiles of 150 selected human ncRNAs. Potential ncRNAs were selected from 5489 transcripts annotated in the H-InvDB2.2, updated in 2005 , by several criteria. The resultant 150 ncRNAs were employed for expression profiling in 11 human tissues. Our RT-qPCR and Northern blot hybridizations revealed that the majority of human ncRNA were expressed in one or a few tissues at relatively low levels, suggesting that ncRNAs play specific biological roles in the tissues in which they are expressed.

\section{Materials and Methods}

The dataset of full-length cDNAs from which the ncRNAs are derived

The original dataset of human ncRNAs was selected from the H-InvDB2.2. We extracted 5489 non-protein-coding transcripts from the annotated database. Currently, the updated dataset has been released as H-Inv3. Non-protein-coding transcripts in the previous release are now annotated as hypothetical short proteins, but their IDs and sequences are unaltered. The dataset of the originally selected ncRNAs from H-InvDB2.2 is available in our original ncRNA database (http://www.ncrna.org/ [6]).

Reverse transcription-quantitative polymerase chain reaction ( $R T$-qPCR)

Total RNAs from adult human tissues were purchased from Clontech. Total RNA $(1 \mu \mathrm{g})$ was reverse transcribed using the QuantiTect reverse transcription kit (Qiagen). The primers, designed by Primer3 software (http://www-genome.wi.mit.edu/ftp/distribution/ software/), were purchased from Invitrogen. Aliquots of CDNA were subjected to real time PCR, performed using a LightCycler 480 SYBR Green I Master (Roche Diagnostics) following the manufacturer's protocol. For each reaction, no amplification was observed when reverse transcription was omitted. Information for primers used in this study is available upon request. To estimate relative abundance among ncRNAs, we used the "cross point" (CP) value as a reference. $\mathrm{CP}$ is the point at which the fluorescence of a sample rises above the background fluorescence and is given in number of amplification cycles. The CP of a sample depends on the initial concentration of target DNA in the sample. For some ncRNA targets, we performed absolute quantification analyses in which serial dilutions of an external standard of known concentration were used to create a standard curve. cDNA probes for Northern blot analyses were used as the external standards. 
Northern blot hybridization

A $10 \mu \mathrm{g}$ aliquot of total RNA per lane was separated on a $0.8 \%$ agarose gel containing $2 \%$ formaldehyde and transferred to a positively charged nylon membrane (Hybond N+; Amersham Biosciences). After UV crosslinking, blots were hybridized to ${ }^{32} \mathrm{P}$-labeled riboprobes at $60^{\circ} \mathrm{C}$, overnight, in hybridization buffer (5X SSC, 5X Denhardt solution, 50\% formamide, $1 \%$ SDS and $0.1 \mathrm{mg} / \mathrm{ml}$ denatured salmon sperm DNA). An autoradiographic image was captured and quantified using a Fuji BAS2500 Bio-imaging analyzer. For low abundance ncRNAs, we optimized the hybridization efficiency by using a high specific activity $\left(\sim 2 \times 10^{9} \mathrm{cpm} / \mu \mathrm{g}\right) \mathrm{CDNA}$ probe and by increasing the probe concentration to 10 $\mathrm{ng} / \mathrm{ml}$. Hybridization was performed at $68^{\circ} \mathrm{C}$ in ExpressHyb Hybridization Solution (Clontech) overnight. Blots containing polyA ${ }^{+}$RNA from different human tissues (Human 12-lane MTN Blot or Human MTN Blot II) were purchased from Clontech.

\section{Comparative genomics}

Mouse counterparts of human ncRNAs were identified by sequence comparison and mapped onto the mouse genome. The mapping was performed using the liftOver program in the UCSC genome browser (http://genome.ucsc.edu/cgi-bin/hgGateway [7]) with the following changes in parameters. We lowered the minMatch and minBlocks parameters to 0.01 . Flanking known genes of both query human ncRNA and putative counterpart sequence were withdrawn to validate whether the two genes were aligned in the same order in syntenic regions of each genome. In parallel, human ncRNAs were subjected to BLASTN searches against the FANTOM3 mouse cDNAs database (ftp://fantom.gsc.riken.jp/fantomdb/3.0/). The resultant hits were examined by manual inspection to determine if they were present in a mouse syntenic region.

\section{Results and Discussion}

\section{Selection of ncRNAs from the database}

The selection procedure is summarized in Fig. 1. We used several sets of selection criteria to identify putative ncRNAs, as follows. For the initial selection, the transcript must: 1) have no overlap with other gene transcripts, 2) not be truncated due to errors during cDNA synthesis, and 3) not be a transcript derived from retroposons or transposons. To select such ncRNAs, 5489 transcripts annotated in the H-InvDB2.2 were employed as the starting material. Among these 5489 transcripts, 712 "putative ncRNA" (as defined in $\mathrm{H}$-InvDB) were subjected to the following additional selection criteria. 
First, transcripts composed of $>30 \%$ repetitive elements were excluded. Second, only transcripts possessing a poly (A) signal (AAUAAA or AUUAAA) within approximately $50 \mathrm{bp}$ from the $3^{\prime}$ end of the CDNA were included. Because relatively large numbers of CDNAs in the H-InvDB have poly (A) tail-like sequences at their $3^{\prime}$ termini but lack the poly (A) signal, this selection step is important to exclude truncated CDNA clones. In such cases, the cDNA synthesis may have initiated from an adenine (A)-rich region in the middle of transcripts, resulting in 3'-truncated cDNAs. Third, transcripts that overlap with any other full-length cDNA clones in the H-InvDB were excluded. In case multiple ncRNAs are aligned to the same cluster, we adopt the longest one as a representative clone. This step was done in order to exclude the cDNAs that correspond to a portion of protein-coding genes. Finally, transcripts whose expression was validated by the existence of any EST clones were adopted for "ncRNA candidates". In a second selection proto col, transcripts which had $>70 \%$ sequence homology to mouse transcripts were selected from 2564

"uncharacterized transcripts" (as defined in H-InvDB), and were then subjected to the additional selection criteria described above.

It has been reported that considerable fraction of CDNAs is a product of internal priming from the A-rich region $[8,9]$. We examined whether A-rich sequence is present at the $3^{\prime}$ end of all the ncRNAs in the list. We also examined its presence in the genomic region immediately downstream of all the ncRNAs. The survey resulted in six clones with adjacent A-rich regions. However, four out of these six clones possess typical

polyadenylation signals near their $3^{\prime}$ termini, suggesting that polyA tails were added posttranscriptionally at those positions rather than produced by the internal priming. The remained two clones (HIT000002513 and HIT000018844) are likely to be internally primed, since they lack the typical polyadenylation signals and are followed by consecutive polyA stretches. The resultant $\underline{150}$ ncRNAs are listed in Supplemental Table 1 . These $\underline{150}$ ncRNAs are expected to be intact and independent transcription units, and, thus, provide an appropriate ncRNA pool, free of the controversies described above, for an initial analysis of ncRNAs.

We noticed that a number of cDNAs are located near the 3'end of other protein-coding genes. Although it is possible that they are the $5^{\prime}$ truncated CDNAs derived from the read-through transcripts of the upstream genes, we temporarily adopt these clones as the candidates of "the independent ncRNA" unless they overlap with the upstream cDNA clones. Our preliminary results of RNase protection assay indicated that at least two CDNAs in our list, which aligned close to the upstream protein-coding genes, possess their own $5^{\prime}$ ends, therefore are the independent ncRNAs (data not shown). We cannot exclude the possibility that the selected ncRNAs code for small polypeptides; this 
possibility could be addressed by determining the presence of each transcript in polysomal fractions.

The levels of ncRNAs expressed in HeLa cells were observed to decrease upon treatment with $\alpha$-amanitin for $48 \mathrm{~h}$, indicating that the majority of human ncRNAs are transcribed with RNA polymerase II, as are mRNAs (data not shown). Examination of these $\underline{150}$ ncRNAs revealed that only $\underline{60}$ ncRNAs ( $\underline{40 \%}$ ) are produced from intron-containing genes (Supplemental Table 1). This characteristic of ncRNA genes is distinct from protein-coding genes in which $>90 \%$ are intron-containing genes. In mRNA biogenesis, pre-mRNA splicing is critical to determine the fate of the resultant mRNA as well as to remove introns [10]. These data raise an interesting possibility that the characteristic gene structure influences the intracellular behavior of ncRNAs, which may be distinct from that of mRNAs.

\section{The majority of ncRNAs are expressed in a tissue-specific manner}

To examine the distribution and relative abundance of the selected ncRNAs in human tissues, we used RT-qPCR with 11 adult human tissues. Experiments were repeated at least twice for each primer-pair using template tissue RNAs from different individuals with some exceptions. Representative profiling results are shown in Fig. 2A. We divided tissue-specific expression patterns into three categories: 1) ncRNAs exclusively or predominantly expressed in a single tissue; 2) ncRNAs expressed in a limited number of tissues; and 3) ncRNAs ubiquitously expressed. To quantify expression of ncRNAs, the ncRNAs exclusively or predominantly expressed in a single tissue were scored for the corresponding tissue. Clones expressed in a few tissues were scored only once as being expressed in the tissue with the highest expression, and were not counted for the rest of tissues expressing the clone. Clones ubiquitously expressed in all tissues were scored separately as "ubiquitous".

Of the 150 candidate ncRNAs, we detected expression of $\underline{121}$ ncRNAs in at least one of the tissues tested (Fig. 2B). Forty-four ncRNAs (29\%) were expressed exclusively or predominantly in one tissue, and $\underline{56}$ ncRNAs ( $\underline{38 \%}$ ) were expressed in a small subset of tissues. The rest of the ncRNAs were ubiquitously expressed ( $\underline{21}$ ncRNAs; $14 \%$ ). These data show that $11 \%$ of ncRNAs were expressed in at least one of the 11 tissues examined, and $\underline{67 \%}$ of ncRNAs exhibited a tissue-specific expression pattern. In contrast, no expression was detected for $\underline{29}$ ncRNAs $(\underline{19 \%})$, at least in the 11 tissues we examined and with the PCR primer pairs we used. These data reveal that the majority of human ncRNAs are expressed in a tissue-specific manner, and suggest that the selected ncRNAs play specific physiological roles in distinct tissues rather than housekeeper roles in every tissue. 
The result also excludes the possibility that ncRNA-like transcripts merely originate from genomic DNA contamination and, therefore, are non-functional.

We tabulated the tissue-distribution patterns by classifying each ncRNA with respect to tissue specificity, according to the three categories defined above (Supplemental Table 2). These data are summarized in Fig. 2C. The number of ncRNAs predominantly expressed in testis was conspicuous; testis specific ncRNAs formed the largest subset, containing $\underline{29 \%}$ of all the expressed ncRNAs. The second largest group was those ncRNAs predominantly expressed in brain, which contained $17 \%$ of all the expressed ncRNAs. The ncRNAs specifically expressed in thymus followed with $7 \%$. More than $50 \%$ of ncRNAs belong to these three groups. Similar tendencies were observed in mouse ncRNAs [9], implying that ncRNAs may be involved in complex regulatory mechanism(s) in testis and brain in mammals. Recently, Pollard et al. reported that HAR1, an RNA that has evolved more rapidly in humans than in other mammals, was expressed in a specific region of the neocortex in the developing human brain [11]. It will be of interest to compare the brain-specific ncRNAs from this study with respect to the sequence conservation across various mammalian species. Some ncRNAs specifically expressed in testis have also been

reported in mouse [12]. Furthermore, novel classes of small RNAs (piRNA or rasiRNA) are expressed only in germ line organs in mammals and in Drosophila [13]. Together with small RNA species, testis-specific ncRNAs may impart yet another dimension of gene regulatory mechanisms to the testis.

\section{The majority of ncRNAs are low abundance transcripts}

To confirm the RT-qPCR results, we performed Northern blot analyses. We used riboprobes to verify the transcript orientation because some ncRNA-like transcripts were registered with the opposite orientation in the H-InvDB and, in some cases; the antisense strand was also transcribed. For ncRNAs that exhibited relatively high abundance in RT-qPCR experiments, we detected bands closely corresponding to the registered length in the H-InvDB (Fig. 3: brain, thymus, heart, lung, testis, placenta, skeletal muscle and ubiquitous). We observed additional bands in the blots of the representative ncRNAs for brain, testis, and placenta. Interestingly, the transcript distribution pattern of the representative placenta ncRNA obtained by RT-qPCR analysis (Fig. 2A) was most similar to the distribution of the additional band (the open arrowhead in the panel of Placenta of Fig. 3) rather than the distribution of the expected size band.

ncRNAs whose "cross point" (CP) values in RT-qPCR (see Materials and Methods) were over 30 were undetectable in conventional Northern blot hybridization conditions (e.g. Supplemental Figure upper panel: liver, spleen, kidney, prostate). Therefore, we switched 
to a cDNA probe and employed poly $(A)+$ RNA blots in order to maximize hybridization sensitivity. This change was successful only for the liver specific ncRNA. For the rest of the ncRNAs, changing the probe and/or RNA blot did not enable us to detect hybridization (Supplemental Figure lower panel: spleen, kidney, prostate). This prompted us to perform an absolute quantification of ncRNA transcripts in tissue samples. We estimated the amount of target RNA in $10 \mu \mathrm{g}$ of total tissue RNA, since this amount corresponded to the amount of RNA per lane in the Northern blot gels. The amount of target ncRNA was estimated to be $0.46 \mathrm{fg}$ in prostate $(C P=33), 0.96 \mathrm{fg}$ in kidney $(C P=32), 9.83 \mathrm{fg}$ in liver $(\mathrm{CP}=30)$ and $1.17 \mathrm{pg}$ in skeletal muscle $(\mathrm{CP}=22)$. Thus, the $\mathrm{CP}$ values roughly reflected the actual ncRNA levels.

Our hybridization conditions were able to detect as little as $0.1 \mathrm{pg}$ RNA per lane; therefore, this quantification data was consistent with the Northern hybridization results in which a clear detection threshold existed at approximately CP30. Even for clones where bands were successfully detected, CP values in the RT-qPCR experiments were much higher, and band intensity in Northern blot hybridizations were much lower, than values for abundant mRNAs such as $\beta$-actin $(C P=20)$, or glycerol-3-phosphate dehydrogenase $(C P=17)$ (data not shown). The $C P$ values for the majority of ncRNAs were over 30 . Thus, we concluded that most of the ncRNA candidates are low abundant transcripts. This low abundance may indicate that tissue-specific ncRNA expression is restricted to certain cell types in the tissues. In situ hybridization against human tissue samples would confirm this interesting possibility.

Expression-patterns exhibited by the human ncRNAs are shared between the human and mouse nCRNAs

Our analyses above revealed characteristic expression profiles of human ncRNAs that mirrored the expression profiles of mouse ncRNAs. Using comparative genomics, we assessed the degree of overlap between human ncRNAs and mouse ncRNAs. First, the presence of the syntenic region in the mouse genome was searched by identifying the neighboring genes of each of the 150 ncRNAs in the human genome. The syntenic regions in the mouse genome were mapped for 93 ncRNAs. Second, the presence of any mouse transcripts with significant sequence similarity to the human ncRNAs was searched using the BLASTN program, and $\underline{74}$ ncRNAs were found to correspond to mouse cDNA clones in the FANTOM3 datasets ( $P$-value $=<0.1$ ). Finally, we examined whether the 74 mouse cDNA clones mapped to syntenic regions, and found that $\underline{40}$ mouse cDNAs mapped to syntenic regions of the genome. A data collection of this search is available at our website (http://www.ncrna.org/papers/sasaki/). We concluded that $\underline{40}$ ncRNAs ( $\underline{27 \%}$ of $\underline{150}$ 
ncRNAs) are conserved between human and mouse. We merged the result of this counterpart search with the tissue-specificity profiling data to assess the degree of overlap in expression between human and mouse ncRNA. Fig. 2D shows that human ncRNAs having mouse counterparts exist in most of the tissues examined, with the exception of spleen, liver and placenta. It should be noted that three human ncRNAs for which we did not detect expression did indeed have mouse counterparts, suggesting that they may be transcribed in tissues other than the 11 tissues we examined. We found that a number of brain- or testis-specific human ncRNAs lacked mouse counterparts. This evidence raises the intriguing possibility that these ncRNAs are involved in phenomena specific to humans. Recently, a pattern of tissue specificity similar to human has been reported using microarray analysis of a much larger population of mouse ncRNAs [9]. Our comparative genomic analysis combined with the expression profiling data support the hypothesis that the characteristic expression profile exhibited by human ncRNAs is conserved in the mouse transcriptome despite of low degree of overlap between human and mouse ncRNAs.

In summary, we have identified large numbers of tissue-specific ncRNAs in human, most of which are present as low abundance transcripts. Some ncRNAs are conserved between human and mouse, but the majority of ncRNAs found in human were not present in mouse. Further analysis of individual ncRNAs will reveal important functions in the respective human tissues. Our selected ncRNA collection will provide an important resource for that line of analysis.

\section{Acknowledgments}

We thank K. Watanabe for valuable discussions and encouragement. K. Aoki, M. Shibuya, M. Nagai and M. Togawa are acknowledged for their assistance. This research was supported by the New Energy and Industrial Technology Development Organization (NEDO), a PRESTO program grant from the Japan Science and Technology agency and the Ministry of Education, Culture, Sports, Science, and Technology of Japan.

\section{References}

[1] J.S. Mattick, Nonco ding RNAs: The architects of eukaryotic complexity, EMBO Rep. 211 (2001) 986-991.

[2] K.V. Prasanth and D.L. Spector, Eukaryotic regulatory RNAs: an answer to the 'genome 
complexity' conundrum, Genes and Dev. 21 (2007) 11-42.

[3] R.B. Lanz, N.J. McKenna, S.A. Onate, U. Albrecht, J. Wong, S.Y. Tsai, M-J Tsai and B.W. O'Malley, A steroid receptor coactivator, SRA, functions as an RNA and is present in an SRC-1 complex, Cell 97 (1999) 17-27.

[4] F. Sleutels, R. Zwart and D.P. Barlow, The non-coding Air RNA is required for silencing autosomal imprinted genes, Nature 415 (2002) 810-813.

[5] A.T. Willingham, A.P. Orth, S. Batalov, E.C. Peters, B.G. Wen, P. Aza-Blanc, J.B. Hognesch and P.G. Shult, A strategy for probing the function of noncoding RNAs finds a repressor of NFAT, Science 309 (2005) 1570-1573.

[6] T. Kin, K. Yamada, G. Terai, H. Okida, Y. Yoshinari, Y. Ono, A. Kojima, Y. Kimura, T. Komori and K. Asai, fRNAdb: a platform for mining/annotating functional RNA candidates from non-coding RNA sequences, Nucleic Acids Res. 35 (2007) D145-148.

[7] R.M. Kuhn, D. Karolchik, A.S. Zweig, H. Trumbower, D.J. Thomas, A. Thakkapallayil, C.W. Sugnet, M. Stanke, K.E. Smith, A. Spiel, K.R. Rosenbloom, B. Rhead, B.J. Raney, A. Pohl, J.S. Pedersen, F. Hsu, A.S. Hinrichs, R.A. Harte, M. Diekhans, H. Clawson, G. Bejerano, G.P. Barber, R. Baertsch, D. Haussler and W.J. Kent, The UCSC genome browser database: update 2007, Nucleic Acids Res. 35 (2007) D668-673.

[8] M.Furuno, K.C. Pang, N. Ninomiya, S. Fukuda, M.C. Frith, C. Bult, C. Kai, J. Kawai, P. Carninci, Y. Hayashizaki, J.S. Matick and H. Suzuki, Clusters of internally primed transcripts reveal novel long noncoding RNAs, PLoS Genet. 2 (2006) 537-553.

[9] T. Ravasi, H. Suzuki, K.C. Pang, S. Katayama, M. Furuno, R. Okunishi, S. Fukuda, K. Ru, M.C. Frith, M.M. Gongora, S.M. Grimmond, D.A. Hume, Y. Hayashizaki and J.S. Mattick, Experimental validation of the regulated expression of large numbers of non-coding RNAs from the mouse genome, Genome Res. 16 (2006) 11-19.

[10] H. Le Hir, A. Nott and M.J. Moore, How introns influence and enhance eukaryotic gene expression, Trends Biochem. Sci. 28 (2003) 215-220.

[11] K.S. Pollard, S.R. Salama, N. Lambert, M.-A. Lambot, S. Coppens, J.S. Pedersen, S. 
Katzman, B. King, C. Onodera, A. Siepel, A.D. Kern, C. Dehat, H. Igel, M. Ares P. Vanderhaeghen and D. Haussler, An RNA gene expressed during cortical development evolved rapidly in humans, Nature 443 (2006) 167-172.

[12] Y.S. Cho, N. Iguchi, J. Yang, M.A. Handel and N.B. Hecht, Meiotic messenger RNA and noncoding RNA targets of the RNA-binding protein translin (TSN) in mouse testis, Biol. Reprod. 73 (2005) 840-847.

[13] V.V. Vagin, A. Sigova, C. Li, H. Seitz, V. Gvozdev and P.D. Zamore, A distinct small RNA pathway silences selfish genetic elements in the germline, Science 313 (2006) 305-306.

\section{Figure Legends}

Figure 1

Screening procedure to identify ncRNAs from H-InvDB2.2. Putative ncRNAs and uncharacterized cDNA entries were subjected to manual inspection by several criteria. See text for details.

Figure 2

Expression profile of ncRNA candidates. (A) Tissue-distribution pattern of the representative ncRNAs. Relative abundance among 11 different tissues was examined by RT-qPCR for each ncRNA. Representative ncRNA clones for each of 11 tissues and a clone representing the "ubiquitous" pattern are shown. The abundance in each tissue was normalized and aligned in following order: (from left to right) brain, thymus, heart, lung, liver, spleen, kidney, testis, placenta, prostate, and skeletal muscle. Bars in the histogram were color-coded as follows: black, exclusive or predominant in a single tissue; dark gray, expressed in several tissues; light gray, ubiquitous. See Supplemental Table 3 for ID of representative clones. (B) Summary of RT-qPCR for 150 ncRNAs. The same color-code is used as in the histograms in (A) and white indicates the ncRNAs whose expression was not detected in any of the 11 tissues. The results are tabulated in Supplemental Table 2. (C) The tissue-specific expression pattern of all ncRNAs is summarized. (D) Comparison of tissue specificity of the selected ncRNAs in human and mouse. The results of the human-mouse counterpart search were merged onto the human ncRNA tissue specific distribution histogram shown in (C). The ncRNAs having no mouse counterparts are shown in black in each bar. 
Figure 3

Northern blot analysis of representative ncRNAs. Eight representative clones probed with riboprobes. Total RNAs from 11 tissues and HeLa cells were analyzed. Positions of RNA size markers are shown to the left of each blot. CP value is shown in parentheses after the name of each tissue. Filled arrowheads indicate the bands corresponding to the expected size while open arrowheads indicate additional hybridization bands. Tissue abbreviations: $\mathrm{Br}$; brain, Th; thymus, He; heart, Lu; lung, Li; liver, Sp; spleen, Ki; kidney, Te; testis, Pl; placenta, Pr; prostate, Mu; skeletal muscle. IDs and sizes of ncRNAs are given in Supplemental Table 3. 


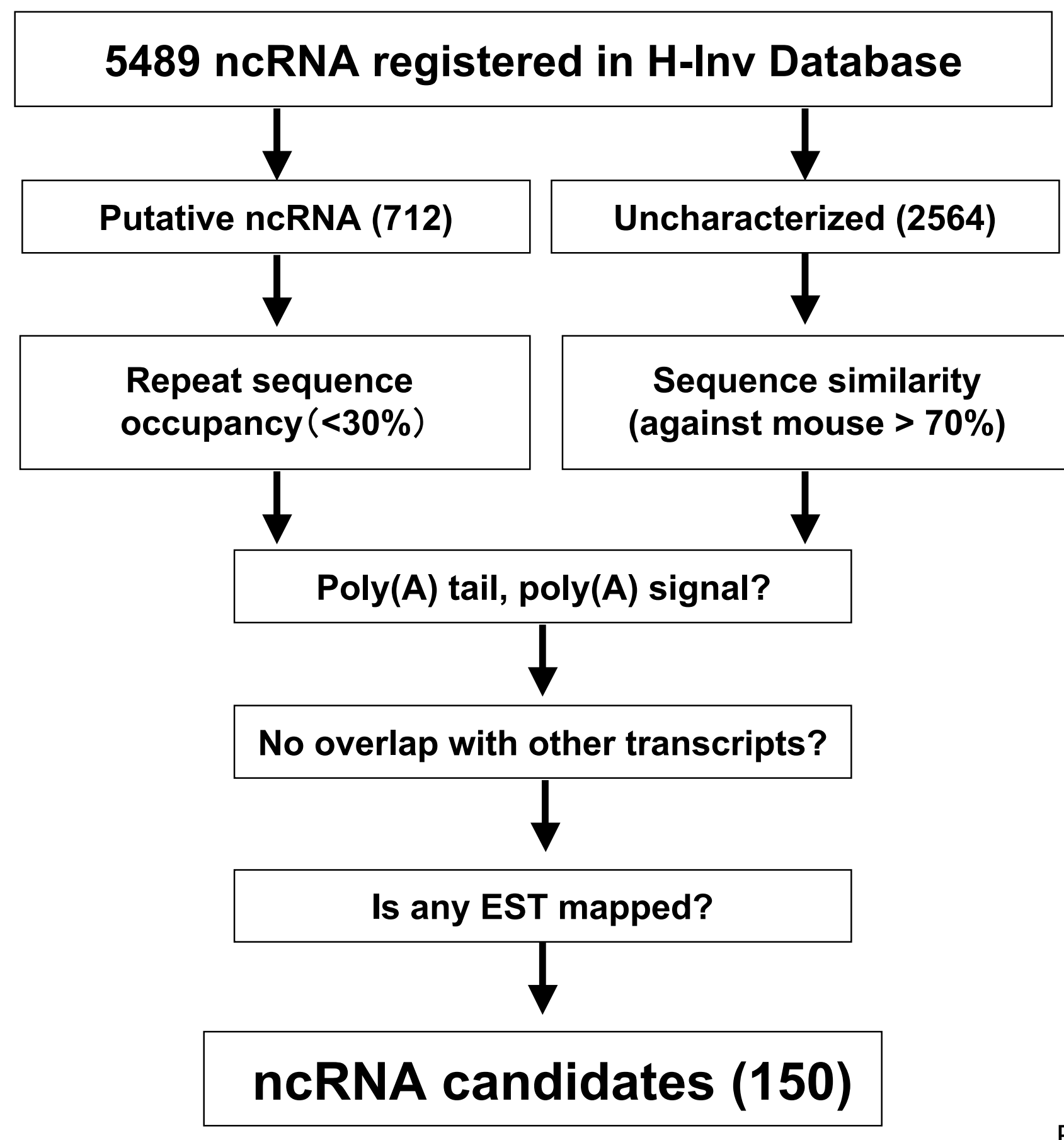

Fig. 1 Sasaki et al. 
A
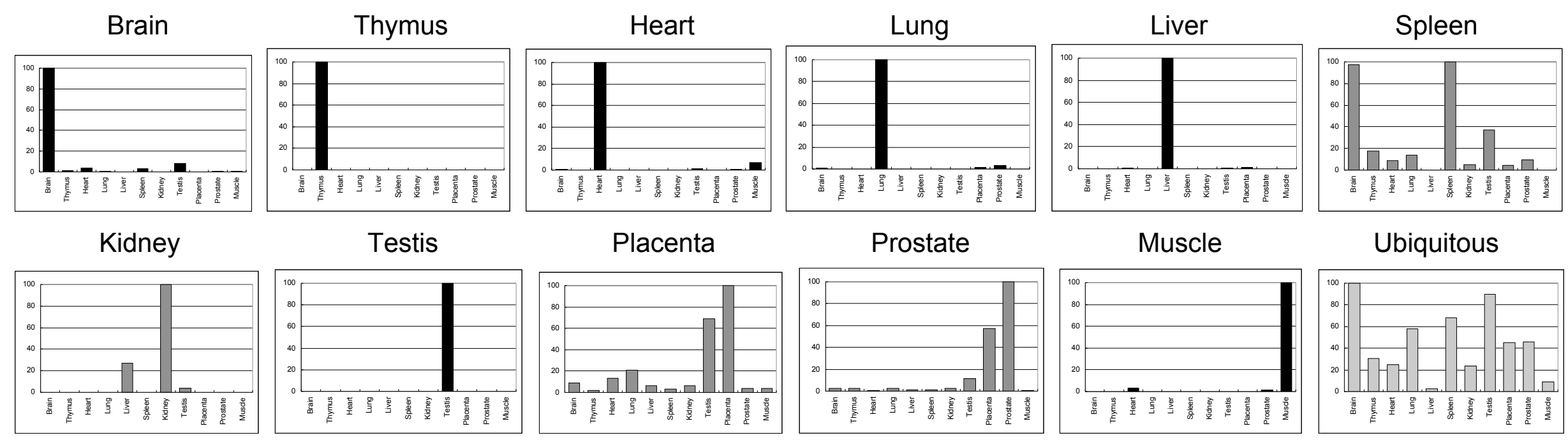

B

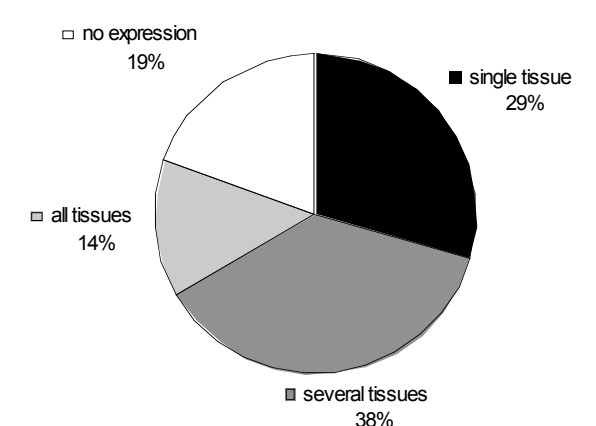

C

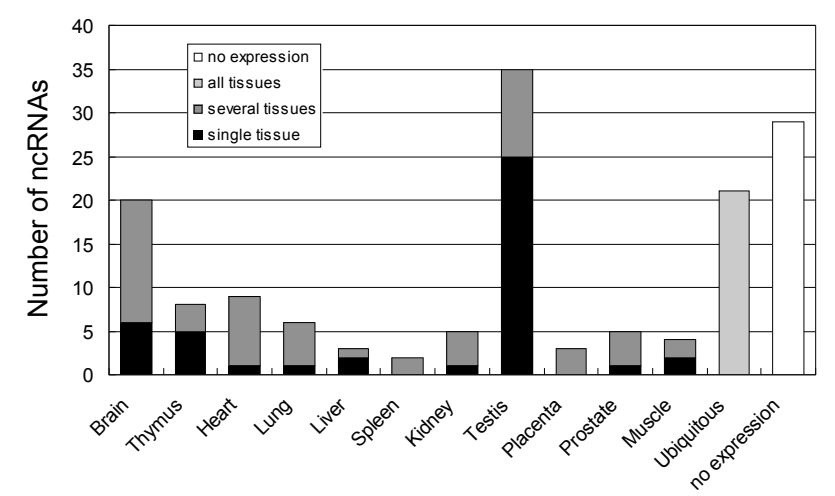

D

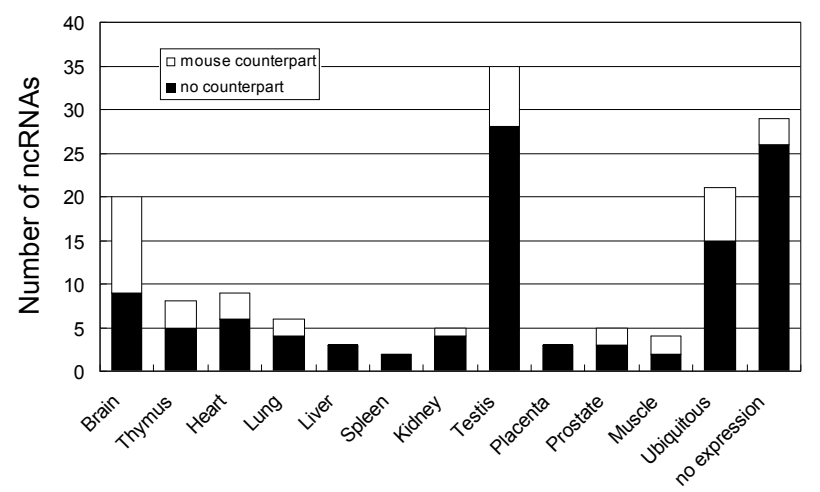

Fig. 2 Sasaki et al. 


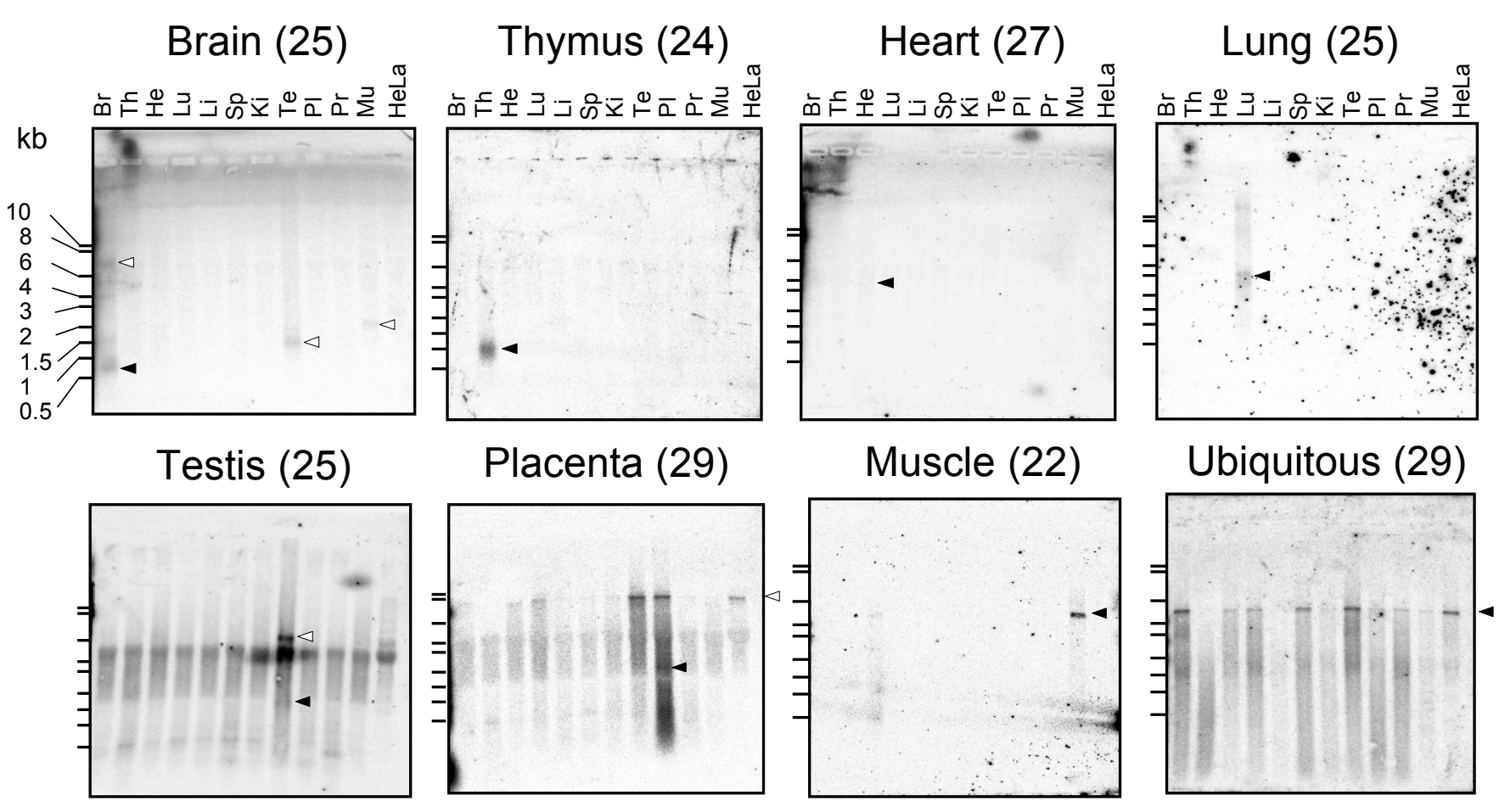

Fig. 3 Sasaki et al. 


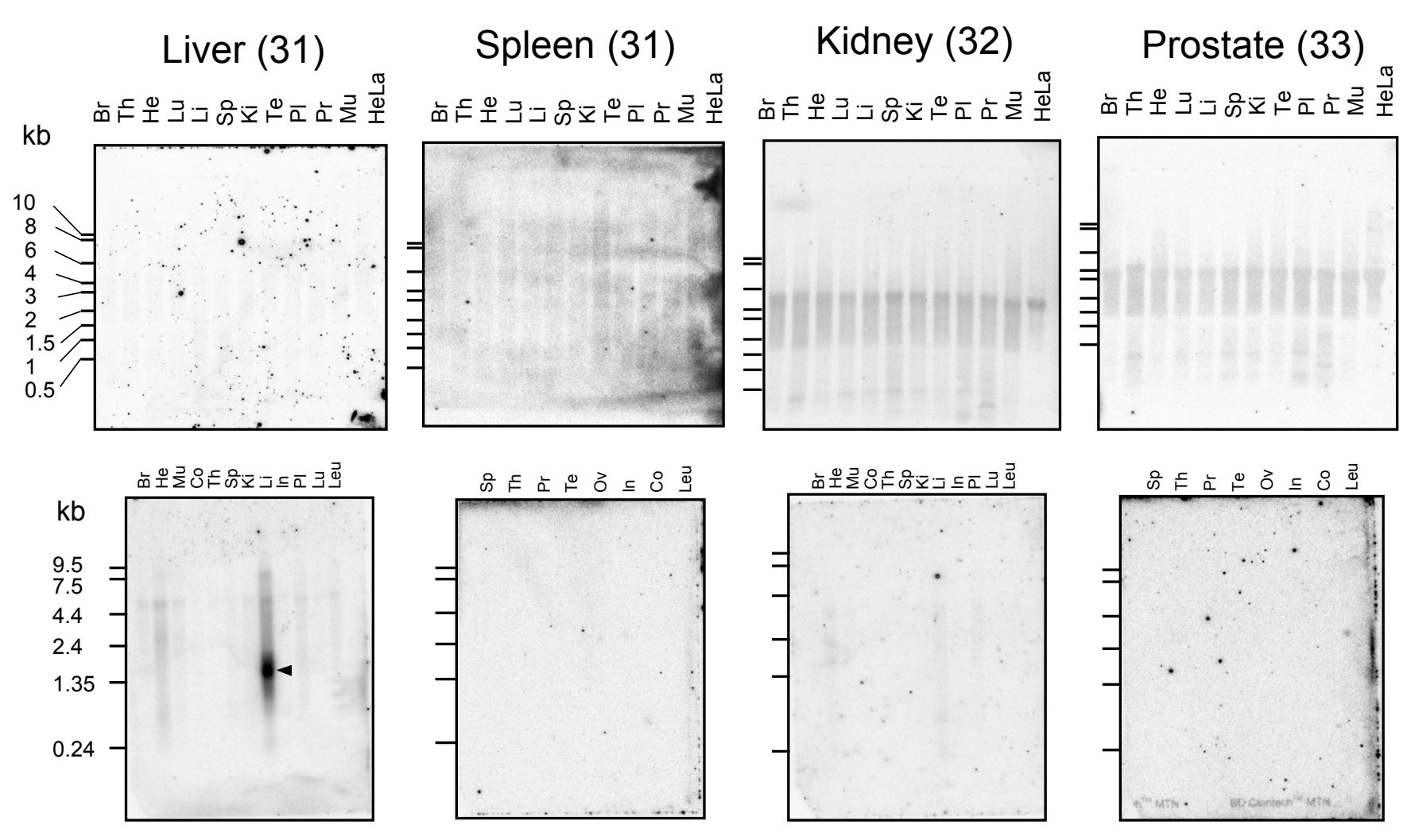

Supplemental Figure Sasaki et al. 


\section{Supplemental Figure Legend}

Supplemental Figure

Northern blot analysis of representative ncRNAs. Extremely rare ncRNAs were hybridized with high specific activity cDNA probes. Positions of RNA size markers are shown to the left of each blot. CP value is shown in parentheses after the name of each tissue. Upper panels. Blots are the same as in Fig. 3. Lower panels. Commercially obtained poly $(A)+$ RNA blots from 12 human tissues were probed with ncRNAs representing liver and kidney. Commercially obtained poly $(A)+$ blots from 8 tissues were probed with ncRNAs representing spleen and prostate. Tissue abbreviations: $\mathrm{Br}$; brain, $\mathrm{Co}$; colon, He; heart, In; small intestine, Ki; kidney, Leu; peripheral blood leukocyte, Li; liver, Lu; lung, Mu; skeletal muscle, Ov; ovary, Pl; placenta, Pr; prostate, $\mathrm{Sp}$; spleen, Te; testis, Th; thymus. IDs and sizes of ncRNAs are given in Supplemental Table 3. 
Supplemental Table 1. A list of 150 selected human ncRNA candidates

\begin{tabular}{|c|c|c|c|c|c|c|c|c|c|}
\hline ncRNA candidate & Accession No. & Category*1 & Conservation & intron & $\mathrm{EST}^{* 2}$ & cDNA library & H-Angel & Tissue-specificity & ncRNA candidates \\
\hline HIT000002513 & AK000038 & - & & - & $\mathrm{A}$ & adipose tissue & eye, placenta, lung & $*^{3}$ lung, liver & HIT000002513 \\
\hline HIT000003532 & AK001058 & + & & - & A & whole embryo, mainly head & placenta & lung, placenta, heart & HIT000003532 \\
\hline HIT000004025 & AK001551 & + & & + & $\mathrm{B}$ & NA & liver & & HIT000004025 \\
\hline HIT000004056 & AK001582 & + & & + & $\mathrm{B}$ & NA & cerebrum & & HIT000004056 \\
\hline HIT000004377 & AK001903 & unch & + & - & A & placenta & ubiquitous & & HIT000004377 \\
\hline HIT000004808 & AK021534 & unch & + & - & A & placenta & lung or ubiquitous & testis, lung, brain, thymus, prostate & HIT000004808 \\
\hline HIT000006301 & AK023027 & unch & + & + & A & NA & cerebrum & brain, testis & HIT000006301 \\
\hline HIT000007366-> & AK024092 & unch & + & - & $\mathrm{A}$ & whole embryo, mainly head & lung or ubiquitous & $*^{3}$ lung, brain, thymus, testis, heart & HIT000007366->92364 \\
\hline HIT000007377 & AK024103 & unch & + & - & A & whole embryo, mainly head & ubiquitous & $*^{3}$ muscle & HIT000007377 \\
\hline HIT000007630 & AK024356 & unch & + & - & A & placenta & spine, breast & heart, muscle, brain & HIT000007630 \\
\hline HIT000008220 & AK024946 & + & & - & A & colon & ubiquitous & heart, brain & HIT000008220 \\
\hline HIT000009660 & AK026386 & + & & + & $\mathrm{C}$ & human small intestine & cerebrum & testis & HIT000009660 \\
\hline HIT000009690 & $\underline{\mathrm{AK} 026416}$ & + & & + & $\mathrm{C}$ & ileal mucosa & ubiquitous & kidney, liver & HIT000009690 \\
\hline HIT000010157 & AK026883 & + & & + & $\mathrm{A}$ & NA & ubiquitous & $*^{3}$ lung, testis, spleen & HIT000010157 \\
\hline HIT000010473 & $\underline{\text { AK027199 }}$ & unch & + & - & $\mathrm{A}$ & lung & ubiquitous & brain, testis, lung, placenta, thymus & HIT000010473 \\
\hline HIT000010507 & AK027233 & + & & + & $\mathrm{C}$ & lung & $?$ & lung, testis & HIT000010507 \\
\hline HIT000011439 & AK054825 & + & & + & $\mathrm{C}$ & cerebellum & cerebrum, colon, kidney & heart, brain & HIT000011439 \\
\hline HIT000011547 & AK054933 & + & & - & $\mathrm{B}$ & cerebellum & adipose, breast & brain, thymus & HIT000011547 \\
\hline HIT000011622 & AK055008 & + & & - & $\mathrm{C}$ & cerebellum & cerebrum & muscle, brain, heart & HIT000011622 \\
\hline HIT000011636 & AK055022 & + & & - & A & cerebellum & pancreas & thymus, lung, spleen & HIT000011636 \\
\hline HIT000011828 & AK055214 & unch & + & - & $\mathrm{A}$ & NA & ubiquitous & prostate, testis, skeletal & HIT000011828 \\
\hline HIT000011885 & AK055271 & + & & - & $\mathrm{C}$ & brain & $?$ & testis, heart, brain & HIT000011885 \\
\hline HIT000011965 & AK055351 & + & & + & $\mathrm{B}$ & brain & colon & & HIT000011965 \\
\hline HIT000012073 & AK055459 & + & & + & $\mathrm{A}$ & brain & breast & brain, prostate, lung & HIT000012073 \\
\hline HIT000012128 & AK055514 & + & & + & $B$ & NA & testis & testis & HIT000012128 \\
\hline HIT000012261 & AK055647 & + & & - & A & NA & cerebrum & brain, testis & HIT000012261 \\
\hline HIT000012319 & AK055705 & + & & - & $\mathrm{B}$ & NA & cerebrum & & HIT000012319 \\
\hline HIT000012733 & AK056119 & + & & + & $\mathrm{B}$ & NA & colon, kidney & testis, (lung, prostate) & HIT000012733 \\
\hline HIT000012825 & AK056211 & + & & + & $\mathrm{C}$ & NA & ovary & $*^{3}$ testis & HIT000012825 \\
\hline HIT000012901 & AK056287 & + & & + & $\mathrm{C}$ & NA & $?$ & & HIT000012901 \\
\hline \begin{tabular}{|l|} 
HIT000013099 \\
\end{tabular} & AK056485 & + & & + & $\mathrm{C}$ & NA & $?$ & & HIT000013099 \\
\hline HIT000013398 & AK056784 & + & & + & $\mathrm{A}$ & placenta & heart & placenta, brain, lung & HIT000013398 \\
\hline \begin{tabular}{|l|} 
HITO000013588 \\
\end{tabular} & AK056974 & unch & + & - & $\mathrm{A}$ & skeletal muscle & ubiquitous & lung, brain, testis, heart, placenta & HIT000013588 \\
\hline HIT000013730-> & AK057116 & + & & + & A & spleen & ? & muscle, testis, heart, thymus, brain & HIT000013730->25131 \\
\hline HIT000013840 & AK057226 & + & & - & $\mathrm{C}$ & testis & $?$ & & HIT000013840 \\
\hline HIT000013867 & AK057253 & + & & + & $\mathrm{C}$ & testis & $?$ & testis & HIT000013867 \\
\hline HIT000013911 & AK057297 & + & & + & $\mathrm{C}$ & testis & $?$ & & HIT000013911 \\
\hline HIT000013965 & AK057351 & + & & + & $\mathrm{C}$ & testis & $?$ & testis & HIT000013965 \\
\hline HIT000014168 & AK057554 & + & & + & $\mathrm{B}$ & thymus & $?$ & thymus & HIT000014168 \\
\hline HIT000014470 & AK057863 & + & & + & A & brain & cerebrum & brain, (testis, heart) & HIT000014470 \\
\hline
\end{tabular}




\begin{tabular}{|c|c|c|c|c|c|c|c|c|c|}
\hline HIT000014545 & AK057938 & + & & - & $\mathrm{C}$ & NA & testis & & HIT000014545 \\
\hline HIT000015843 & AK090976 & + & & - & $\mathrm{C}$ & amygdala & liver & testis & HIT000015843 \\
\hline HIT000016553 & AK091686 & + & & - & $\mathrm{B}$ & brain & cerebrum & spleen, brain & HIT000016553 \\
\hline HIT000016555 & AK091688 & + & & + & A & brain & placenta & brain, testis, prostate & HIT000016555 \\
\hline HIT000016590 & AK091723 & unch & + & - & A & NA & retina & ${ }^{* 3}$ brain & HIT000016590 \\
\hline HIT000016701 & AK091834 & + & & + & $A$ & lung & $?$ & Lung, (prostate) & HIT000016701 \\
\hline HIT000017066 & AK092199 & + & & + & $\mathrm{B}$ & NA & brain stem & testis & HIT000017066 \\
\hline HIT000017408 & AK092541 & + & & + & $\mathrm{B}$ & prostate & $?$ & ${ }^{* 3}$ testis, (kidney, lung) & HIT000017408 \\
\hline HIT000017445 & AK092578 & + & & + & A & prostate & $?$ & ${ }^{* 3}$ kidney & HIT000017445 \\
\hline HIT000017869 & AK093002 & + & & + & $A$ & spleen & $?$ & $*^{3}$ prostate & HIT000017869 \\
\hline HIT000018283 & AK093416 & + & & + & $A$ & testis & testis, placenta & testis, lung, placenta & HIT000018283 \\
\hline HIT000018359 & AK093492 & + & & + & $\mathrm{C}$ & testis & ubiquitous & testis, kidney & HIT000018359 \\
\hline HIT000018388 & AK093521 & + & & - & $\mathrm{A}$ & testis & cerebrum, placenta, muscle & thymus & HIT000018388 \\
\hline \begin{tabular}{|l|} 
HIT000018698 \\
\end{tabular} & AK093832 & unch & + & - & $\mathrm{A}$ & trachea & ubiquitous & heart, lung, brain & HIT000018698 \\
\hline HIT000018839 & AK093982 & unch & + & - & A & uterus & kidney, artery/aorta, prostate, muscle & & HIT000018839 \\
\hline HIT000018844 & AK093987 & + & & $?$ & $\mathrm{~B}$ & uterus & prostate, colon & prostate, placenta & HIT000018844 \\
\hline HIT000019009 & AK094154 & + & & + & $\mathrm{C}$ & NA & muscle & placenta, testis, brain & HIT000019009 \\
\hline HIT000019317 & AK094462 & + & & + & $\mathrm{C}$ & cerebellum & cerebellum & brain, kidney & HIT000019317 \\
\hline HIT000019431 & AK094576 & + & & + & $\mathrm{B}$ & amygdala & $?$ & testis, (lung, brain) & HIT000019431 \\
\hline HIT000019460 & AK094605 & - & - & - & $\mathrm{C}$ & amygdala & $?$ & & HIT000019460 \\
\hline HIT000019599 & AK094744 & + & & - & A & brain & cerebrum, kidney & brain, kidney, spleen & HIT000019599 \\
\hline HIT000019668 & AK094813 & + & . & - & $\mathrm{B}$ & brain & muscle & prostate, testis, brain & HIT000019668 \\
\hline HIT000019901 & AK095046 & - & + & - & $A$ & hippocampus & cerebrum, stomach & brain & HIT000019901 \\
\hline HIT000020054 & AK095199 & + & + & + & $\mathrm{B}$ & subthalamic nucleus & cerebrum, breast & & HIT000020054 \\
\hline HIT000020137 & AK095282 & + & & + & $\mathrm{B}$ & tongue, tumor tissue & artery/aorta & & HIT000020137 \\
\hline HIT000020163 & AK095308 & + & & - & $\mathrm{B}$ & tongue, tumor tissue & testis, muscle & & HIT000020163 \\
\hline HIT000020554 & AK095699 & + & & + & $\mathrm{B}$ & brain & $?$ & testis & HIT000020554 \\
\hline HIT000020699 & AK095844 & + & & - & $\mathrm{B}$ & NA & $?$ & brain & HIT000020699 \\
\hline HIT000020920 & AK096065 & + & & - & $\mathrm{A}$ & kidney & pineal gland & testis & HIT000020920 \\
\hline HIT000020989 & AK096134 & + & & + & $\mathrm{B}$ & liver & liver & liver, kidney & HIT000020989 \\
\hline HIT000020998 & AK096143 & + & & + & $\mathrm{A}$ & liver & breast, liver & liver & HIT000020998 \\
\hline HIT000021556 & $\overline{\text { AK096701 }}$ & - & - & - & $\mathrm{A}$ & pericardium & ubiquitous & heart, muscle & HIT000021556 \\
\hline HIT000021635 & AK096780 & + & & - & $\mathrm{C}$ & prostate & lung, kidney, pancreas & $\star^{3}$ testis, kidney & HIT000021635 \\
\hline HIT000021759-> & AK096904 & unch & + & & & skeletal muscle & ubiquitous & muscle & HIT000021759->45761 \\
\hline HIT000022361 & AK097507 & + & & - & $\mathrm{C}$ & testis & $?$ & testis & HIT000022361 \\
\hline HIT000022501 & AK097647 & + & & - & $\mathrm{B}$ & testis & $?$ & $\star^{3}$ testis, (thymus ) & HIT000022501 \\
\hline HIT000022977 & AK098142 & + & & - & A & trachea & adipose, lung, cerebrum & lung, brain, heart, spleen, brain, placenta, heal & HIT000022977 \\
\hline HIT000023168 & AK098338 & unch & + & & & uterus & & thymus & HIT000023168 \\
\hline HIT000023427 & AK098597 & + & & + & $\mathrm{B}$ & testis & cerebrum, breast & ${ }^{* 3}$ thymus, spleen, kidney, brain, lung, & HIT000023427 \\
\hline HIT000023486 & AK098656 & + & & + & $\mathrm{B}$ & testis & $?$ & & HIT000023486 \\
\hline HIT000023747 & AL049321 & unch & + & & & brain & ubiquitous & testis, heart, lung & HIT000023747 \\
\hline HIT000024044 & AL050166 & - & - & - & A & uterus & ubiquitous & lung, brain & HIT000024044 \\
\hline HIT000024203 & $\overline{\mathrm{AL080082}}$ & + & + & - & $\mathrm{B}$ & brain & placenta & thymus, testis, prostate & HIT000024203 \\
\hline HIT000024337 & AL080216 & unch & + & & & uterus & ubiquitous & testis, lung, heart, brain, thymus & HIT000024337 \\
\hline
\end{tabular}




\begin{tabular}{|c|c|c|c|c|c|c|c|c|c|}
\hline HIT000024429 & AL110163 & unch & + & - & $\mathrm{A}$ & uterus & cerebrum & testis, heart, brain, lung, placenta, heart, lung & HIT000024429 \\
\hline HIT000024446 & AL110180 & unch & + & - & A & brain & colon, liver & testis, lung, brain, prostate, spleen & HIT000024446 \\
\hline HIT000024455 & AL110189 & - & + & - & $\mathrm{C}$ & testis & $?$ & & HIT000024455 \\
\hline HIT000024721 & AL117559 & + & & - & $\mathrm{B}$ & kidney & cerebrum & $*^{3}$ thymus, (brain) & HIT000024721 \\
\hline HIT000024815 & AL117653 & unch & + & & & uterus & & heart, muslce & HIT000024815 \\
\hline HIT000025044 & AL133577 & unch & + & - & A & testis & ubiquitous & testis & HIT000025044 \\
\hline HIT000025071 & $\overline{\mathrm{AL} 133604}$ & + & & - & $\mathrm{C}$ & testis & muscle & testis & HIT000025071 \\
\hline HIT000025686 & AL137398 & + & & - & $\mathrm{B}$ & testis & testis & testis & HIT000025686 \\
\hline HIT000025786 & AL137498 & unch & + & 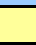 & & amygdala & ubiquitous & ${ }^{3}$ brain, kidney & HIT000025786 \\
\hline HIT000026049 & AL137761 & + & & - & A & uterus & ubiquitous & testis, kidney, prostate, lung ,brain & HIT000026049 \\
\hline HIT000026243 & AL162073 & + & & - & $\mathrm{A}$ & melanoma & ubiquitous & lung, prostate, placenta, muscle, testis & HIT000026243 \\
\hline HIT000027034 & AL832122 & + & + & - & $\mathrm{A}$ & cervix & prostate, cerebrum, muscle & testis, (thymus) & HIT000027034 \\
\hline HIT000027048 & AL832136 & + & + & - & $\mathrm{B}$ & cervix & endocrine, exocrine & ${ }^{3}$ thymus, spleen & HIT000027048 \\
\hline HIT000027165 & AL832253 & + & & - & $\mathrm{B}$ & lymph node & muscle & & HIT000027165 \\
\hline HIT000027207 & AL832295 & + & & + & C? & lymph node & ubiquitous & & HIT000027207 \\
\hline HIT000027585 & AL832673 & unch & + & & & adipose & ubiquitous & brain, testis, spleen, lung, prostate & HIT000027585 \\
\hline HIT000027821 $\rightarrow$ & AL832909 & unch & + & - & A & melanoma & ubiquitous & testis, heart, brain & HIT000027821 \\
\hline HIT000028053 & AL833141 & unch & + & & & heart & peripheral nerve, pancreas & muscle, heart, brain & HIT000028053 \\
\hline HIT000028152 & AL833240 & + & & - & A & amygdala & lung, cerebrum & brain, (lung) & HIT000028152 \\
\hline HIT000028245 & $\overline{\mathrm{AL833333}}$ & + & & + & $\mathrm{C}$ & testis & testis, cerebrum & testis & HIT000028245 \\
\hline HIT000042993 & AK123120 & + & & + & $\mathrm{B}$ & cerebellum & $?$ & testis, (prostate, brain) & HIT000042993 \\
\hline HIT000043067 & AK123194 & + & & + & $\mathrm{B}$ & cerebellum & breast & testis, brain, prostate & HIT000043067 \\
\hline HIT000043322 & AK123449 & + & & - & $A$ & subthalamic nucleus & cerebrum, bone & & HIT000043322 \\
\hline HIT000043631 & AK123758 & + & & + & $\mathrm{B}$ & NA & $?$ & & HIT000043631 \\
\hline HIT000043639 & AK123766 & + & & + & $\mathrm{B}$ & NA & $?$ & prostate, heart, lung & HIT000043639 \\
\hline HIT000043674 & AK123801 & + & & + & $\mathrm{B}$ & ovary & $?$ & spleen, testis & HIT000043674 \\
\hline HIT000044126 & AK124253 & unch & + & & & kidney, tumor tissue & cerebrum, ovary & thymus, (brain) & HIT000044126 \\
\hline HIT000044150 & AK124277 & + & & + & $\mathrm{C}$ & liver, tumor tissue & liver & liver & HIT000044150 \\
\hline HIT000044154 & AK124281 & + & & - & $\mathrm{A}$ & liver, tumor tissue & breast & & HIT000044154 \\
\hline HIT000044215 & AK124342 & + & & + & $\mathrm{C}$ & uterus & $?$ & brain, testis, muscle & HIT000044215 \\
\hline HIT000044222 & AK124349 & + & & + & $\mathrm{C}$ & uterus & colon, liver, lung & & HIT000044222 \\
\hline HIT000044272-> & AK124399 & + & & + & A & bladder & ? & ${ }^{* 3}$ thymus, testis, kidney, spleen, heart & HIT000044272 \\
\hline HIT000044649 & AK124776 & + & & - & $\mathrm{A}$ & brain & cerebrum, skin & brain, kidney & HIT000044649 \\
\hline HIT000044812 & AK124939 & + & & - & $\mathrm{A}$ & subthalamic nucleus & $?$ & brain, heart, liver & HIT000044812 \\
\hline HIT000044831 & AK124958 & + & & - & $\mathrm{A}$ & subthalamic nucleus & ubiquitous & brain, prostate, lung & HIT000044831 \\
\hline HIT000044861 & AK124988 & + & & - & $\mathrm{C}$ & thalamus & eye, muscle & & HIT000044861 \\
\hline HIT000044963 & AK125090 & + & & - & $\mathrm{A}$ & tongue, tumor tissue & artery/aorta, placenta, colon & placenta, testis, lung & HIT000044963 \\
\hline HIT000045023 & $\overline{\mathrm{AK125150}}$ & unch & + & - & $\mathrm{A}$ & brain & ubiquitous & brain, thymus & HIT000045023 \\
\hline HIT000045537 & AK125664 & + & & - & A & synovial membrane tissue from & ubiquitous & brain, testis, kidney & HIT000045537 \\
\hline HIT000046198 & AK126325 & + & & + & $\mathrm{A}$ & trachea & cerebrum, lung & & HIT000046198 \\
\hline HIT000046322 & AK126449 & + & & $?$ & A & uterus & $?$ & lung, testis, kidney, muscle, placenta & HIT000046322 \\
\hline HIT000047747 & AK127874 & + & & + & $\mathrm{C}$ & prostate & cerebrum, breast & testis, (brain) & HIT000047747 \\
\hline HIT000048359 & AK128486 & + & & + & $\mathrm{A}$ & trachea & $?$ & brain, (lung) & HIT000048359 \\
\hline HIT000048747 & $\overline{\text { AK128874 }}$ & + & & + & $\mathrm{A}$ & hippocampus & $?$ & brain, spleen, liver, placenta, testis & HIT000048747 \\
\hline
\end{tabular}




\begin{tabular}{|c|c|c|c|c|c|c|c|c|c|}
\hline HIT000048811 & AK129540 & + & & - & $\mathrm{C}$ & pancreas & breast & $\star^{3}$ testis & HIT000048811 \\
\hline HIT000049265 & AK129994 & + & & + & C & kidney & colon & & HIT000049265 \\
\hline HIT000049320 & AK130049 & - & + & - & $\mathrm{A}$ & kidney & artery/aorta, vein, placenta, muscle, breast & kidney, testis, heart, lung & HIT000049320 \\
\hline HIT000049338 & AK130067 & + & & - & $\mathrm{A}$ & lung & brain stem & heart, lung, kidney, placenta & HIT000049338 \\
\hline HIT000049924 & AK130653 & unch & + & & & spleen & ubiquitous & testis, thymus, lung, heart, brain & HIT000049924 \\
\hline HIT000050135 & AK130864 & + & & + & A & testis & artery/aorta, muscle & testis & HIT000050135 \\
\hline HIT000050297 & AK131026 & + & & + & $\mathrm{B}$ & testis & ubiquitous & & HIT000050297 \\
\hline HIT000050965 & BC029043 & + & & - & $\mathrm{B}$ & brain & $?$ & & HIT000050965 \\
\hline HIT000051024 & BC031235 & + & & + & B & testis & ubiquitous & $*^{3}$ testis & HIT000051024 \\
\hline HIT000051342 & BC035189 & + & & - & $\mathrm{B}$ & testis & ubiquitous & $*^{3}$ testis & HIT000051342 \\
\hline HIT000054382 & BX537597 & unch & + & & & human fetal kidney & $?$ & testis, thymus, lung, heart, brain & HIT000054382 \\
\hline HIT000054391 & BX537606 & + & & - & A & human rectal tumor & bone & & HIT000054391 \\
\hline HIT000054535 & BX537750 & unch & + & & & retina & ubiquitous & thymus, testis, lung, heart, brain & HIT000054535 \\
\hline HIT000054547 & BX537762 & unch & + & - & $\mathrm{A}$ & liver & $?$ & testis, heart, lung & HIT000054547 \\
\hline HIT000054926 & BX538144 & - & - & - & A & fetal kidney & cerebrum, adipose, colon & testis, heart, brain, kidney, thymus & HIT000054926 \\
\hline HIT000055032 & BX538250 & + & & + & $\mathrm{A}$ & testis & heart & testis, brain, thymus & HIT000055032 \\
\hline HIT000055119 & BX538337 & unch & + & & & rectum tumor & ? & testis, heart & HIT000055119 \\
\hline HIT000055794 & BX647147 & - & + & - & $\mathrm{A}$ & human uterine endothelium prim & cerebrum, skin, testis, muscle, kidney & heart, testis, brain & HIT000055794 \\
\hline HIT000056511 & BX647864 & + & & + & $\mathrm{B}$ & adipose & bladder, cerebrum & kidney, testis & HIT000056511 \\
\hline HIT000056687 & BX648040 & + & & - & $\mathrm{C}$ & liver & muscle & heart, muscle & HIT000056687 \\
\hline HIT000057296 & BX648649 & + & & - & $\mathrm{C}$ & fetal kidney & lung, cerebrum & kidney, heart, lung & HIT000057296 \\
\hline HIT000057775 & BX649128 & unch & + & - & A & amygdala & ubiquitous & ${ }^{*}{ }^{3}$ brain, thymus, spleen, lung, prostate & HIT000057775 \\
\hline
\end{tabular}

*1Category: '+; putative ncRNA, unch; uncharacterized ncRNA, '-; all others

*2 No. of supported ESTs: A; >10, B; 5-9, C; 1-4

*3Result of single experiment

*4A-rich region: to be met following two criteria: 1) a polyA tract consecutive of at least seven nucleotides, 2) eight As out of ten nucleotides immediately downstream of 3 '-end

key: $\quad$ testis, (brain)

heart, testis, brain

predominantly expressed in one tissue (i.e., testis), with minor expression elsewhere (i.e., brain

testis, prostate, liver, lung, heart

expressed in limited number of tissues, i.e., heart, testis, and brain

ubiquitously expressed, only top 5 tissues are listed

no expression was observed in 11 tissues examined 


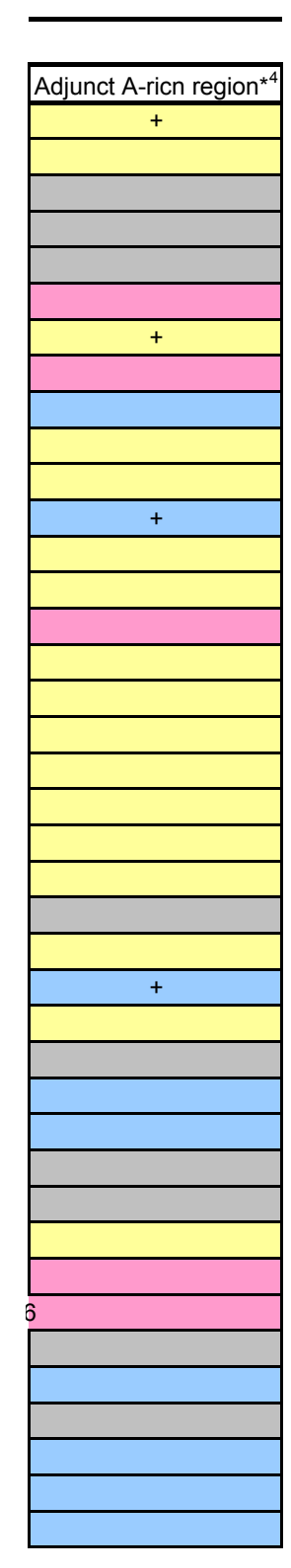




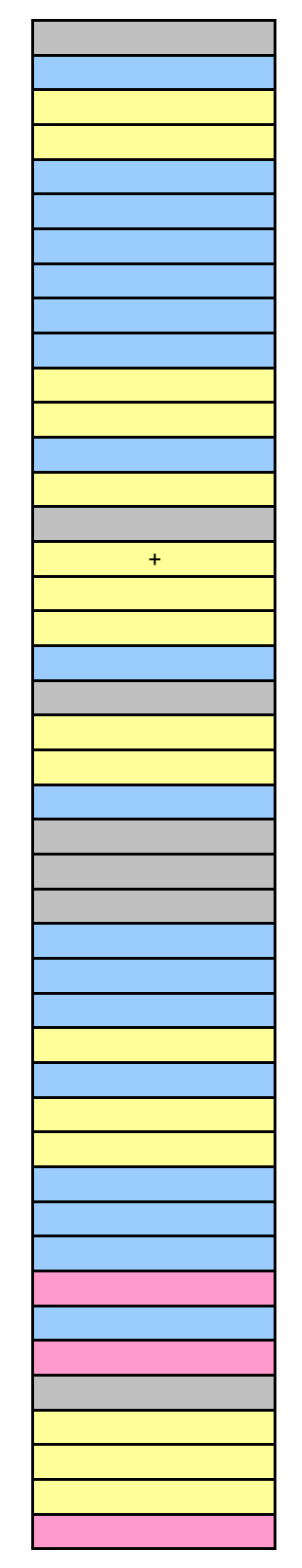




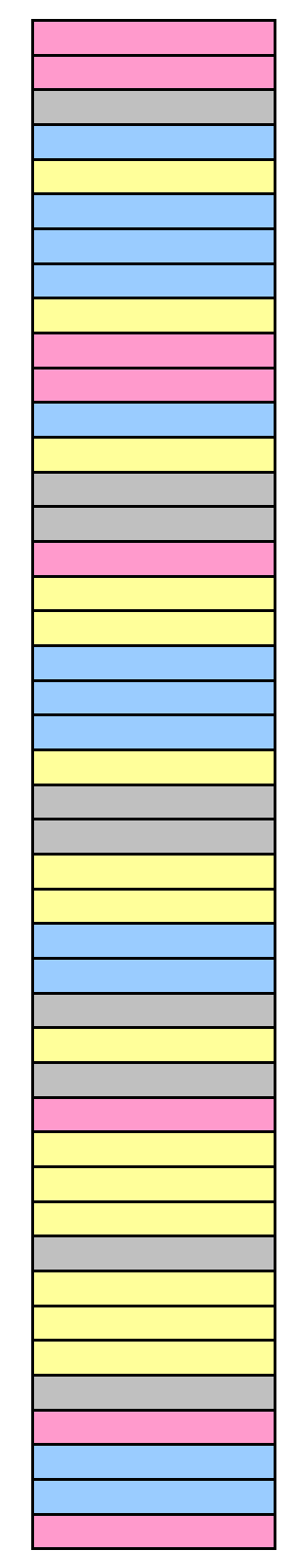




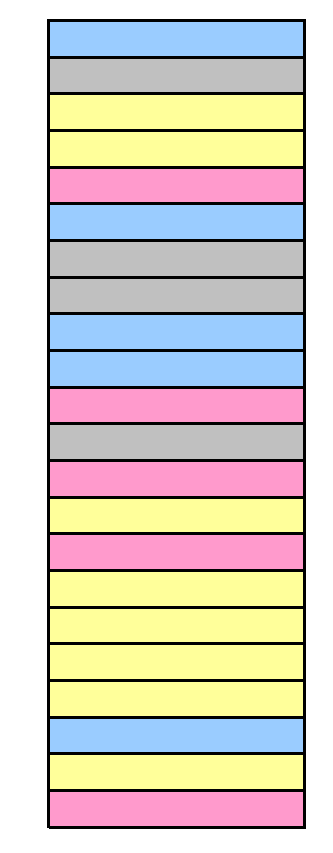


Supplemental Table 2. ncRNAs that exhibited tissue-specific expression patterns.

\begin{tabular}{|c|c|c|c|c|c|c|c|c|c|c|c|c|c|}
\hline & Brain & Thymus & Heart & Lung & Liver & Spleen & Kidney & Testis & Placenta & Prostate & Muscle & \multicolumn{2}{|c|}{ Ubiquitous no expression } \\
\hline 1 & 6301 & 11636 & 7630 & 2513 & 20989 & 16553 & 9690 & 9660 & 13398 & 11828 & 7377 & 4808 & 4025 \\
\hline 2 & 11547 & 14168 & 8220 & 3532 & 20998 & 43674 & 17445 & 11885 & 19009 & 17869 & 11622 & 7366 & 4056 \\
\hline 3 & 12073 & 18388 & 11439 & 10157 & 44150 & & 49320 & 12128 & 44963 & 18844 & 21759 & 10473 & 4377 \\
\hline 4 & 12261 & 23168 & 18698 & 10507 & & & 56511 & 12733 & & 19668 & 28053 & 13588 & 11965 \\
\hline 5 & 14470 & 24203 & 21556 & 16701 & & & 57296 & 12825 & & 43639 & & 13730 & 12319 \\
\hline 6 & 16555 & 24721 & 24815 & 24044 & & & & 13867 & & & & 22977 & 12901 \\
\hline 7 & 16590 & 27048 & 49338 & & & & & 13965 & & & & 23427 & 13099 \\
\hline 8 & 19317 & 44126 & 55794 & & & & & 15843 & & & & 24337 & 13840 \\
\hline 9 & 19599 & & 56687 & & & & & 17066 & & & & 24429 & 13911 \\
\hline 10 & 19901 & & & & & & & 17408 & & & & 24446 & 14545 \\
\hline 11 & 20699 & & & & & & & 18283 & & & & 26049 & 18839 \\
\hline 12 & 25786 & & & & & & & 18359 & & & & 26243 & 19460 \\
\hline 13 & 28152 & & & & & & & 19431 & & & & 27585 & 20054 \\
\hline 14 & 44215 & & & & & & & 20554 & & & & 44272 & 20137 \\
\hline 15 & 44649 & & & & & & & 20920 & & & & 46322 & 20163 \\
\hline 16 & 44812 & & & & & & & 21635 & & & & 48747 & 23486 \\
\hline 17 & 44831 & & & & & & & 22361 & & & & 49924 & 24455 \\
\hline 18 & 45023 & & & & & & & 22501 & & & & 54382 & 27165 \\
\hline 19 & 45537 & & & & & & & 23747 & & & & 54535 & 27207 \\
\hline 20 & 48359 & & & & & & & 25044 & & & & 54926 & 43322 \\
\hline 21 & & & & & & & & 25071 & & & & 57775 & 43631 \\
\hline 22 & & & & & & & & 25686 & & & & & 44154 \\
\hline 23 & & & & & & & & 27034 & & & & & 44222 \\
\hline 24 & & & & & & & & 27821 & & & & & 44861 \\
\hline 25 & & & & & & & & 28245 & & & & & 46198 \\
\hline 26 & & & & & & & & 42993 & & & & & 49265 \\
\hline 27 & & & & & & & & 43067 & & & & & 50297 \\
\hline 28 & & & & & & & & 47747 & & & & & 50965 \\
\hline 29 & & & & & & & & 48811 & & & & & 54391 \\
\hline 30 & & & & & & & & 50135 & & & & & \\
\hline 31 & & & & & & & & 51024 & & & & & \\
\hline 32 & & & & & & & & 51342 & & & & & \\
\hline 33 & & & & & & & & 54547 & & & & & \\
\hline 34 & & & & & & & & 55032 & & & & & \\
\hline 35 & & & & & & & & 55119 & & & & & \\
\hline
\end{tabular}

40

The last 5-digits of the H-InvDB ID (HIT number) are shown.

Clones exclusively or predominantly expressed in a given tissue are typed in blue.

Clones expressed in a limited number of tissues are typed in black.

Clones used in Northern blot hybridization experiments are highlighted.

Clones having a putative mouse counterpart are typed in bold. 
Supplemental Table 3. The representative ncRNA clones shown in Fig. 2A and used in the Northern blot hybridization analyses.

\begin{tabular}{|c|c|c|c|c|}
\hline Tissue & $H-\ln v$ ID & Accession No. & Length (bp) & $\mathrm{CP}^{* *}$ \\
\hline Brain & HIT000014470 & AK057863 & 816 & 25 \\
\hline Thymus & HIT000014168 & $\overline{\mathrm{AK} 057554}$ & $2596^{*}$ & 24 \\
\hline Heart & HIT000056687 & BX648040 & 3995 & 27 \\
\hline Lung & HIT000016701 & AK091834 & 3093 & 25 \\
\hline Liver & HIT000020998 & $\overline{\text { AK096143 }}$ & 1826 & 31 \\
\hline Spleen & HIT000016553 & $\overline{\text { AK091686 }}$ & 2256 & 31 \\
\hline Kidney & HIT000009690 & AK026416 & 1948 & 32 \\
\hline Testis & HIT000025686 & $\overline{A L 137398}$ & 1921 & 25 \\
\hline Placenta & HIT000044963 & $\overline{\mathrm{AK} 125090}$ & 2408 & 29 \\
\hline Prostate & HIT000018844 & AK093987 & 2283 & 33 \\
\hline Skeletal Muscle & HIT000045761 & AK125888 & 4939 & 22 \\
\hline Ubiquitous & HIT000027585 & AL832673 & 5270 & 29 \\
\hline
\end{tabular}

*: A probe was designed to also detect the abundant $0.5 \mathrm{~kb}$ splice variant.

${ }^{* *} \mathrm{CP}=$ "crossing point" 